\title{
Modelling of switched mode power converters using bond graph
}

\author{
A.C. Umarikar and L. Umanand
}

\begin{abstract}
Modelling of the switched mode power converter (SMPC) involves obtaining the large signal, steady-state and small signal representations. Transfer functions and state equations are used for mathematical representation of the SMPC. Obtaining these dynamic models using a mathematical or graphical approach sometimes lead to loss of an intuitive understanding of the system dynamics. A unified graphical method of modelling SMPC using bond graphs is presented. Only the PWM DC-DC converters are considered. The concept of switched power junctions is used for modelling of switching phenomenon in the SMPC. This approach uses a logical process of graphical reduction, which ultimately results in the state equations of the large signal and small signal AC models of the SMPC including the steady-state equations of the SMPC. This method is applicable to SMPC, in both continuous conduction mode and discontinuous conduction mode The proposed modelling approach is illustrated by a few examples.
\end{abstract}

\section{Introduction}

Switched mode power converters (SMPCs) are in a class of variable structure systems. The topology of the dynamical elements of the system depends on the instantaneous positions of the power switch. Several analytical and graphical methods have been proposed in the literature on mathematical modelling of the SMPCs. Among analytical methods, large signal discrete time models for computer simulation and continuous time models for physical understanding has been proposed [1]. Method of state space averaging has been discussed along with obtaining the small signal model of the switching converter [2]. Graphical methods, like the circuit-averaging method [3] and PWM switch model method $[4,5]$ have also been employed to obtain the averaged large signal model of a converter. The switching flow graph nonlinear modelling technique has been used for modelling of switching converters [6]. This approach is a modification of the signal flow graph method for modelling of switching systems. It unifies the method of obtaining large signal, steady-state and small signal models of the switching converter.

This paper explores the bond graph technique to model switched mode power converters. Modelling of PWM DCDC converters is considered. The concept of switched power junctions [7] is used to model the switching phenomenon in the converters. There is one-to-one correspondence between the physical system and bond graph elements. This model visually indicates the cause and effect relationships between energy variables, which enhance the visual understanding of the system. The bond graph unifies the method of obtaining the large signal, steady-state and small-signal model for switched mode power converter. The large signal, steady-state and small signal models are all derived graphically. Further, the bond graph can handle multidomain systems without sacrificing the ease of deriving the state space representation of physical systems by inspection.

\section{Bond graph modelling and concept of switched power junction}

\subsection{Bond graph overview}

A bond graph is a labelled and directed graphical representation of a physical system. The basis of bond graph modelling is power/energy flow in a system. As energy or power flow is the underlying principle for bond graph modelling, there is seamless integration across multiple domains. As a consequence, different domains (such as electrical, mechanical, thermal, fluid, magnetic etc.) can be represented in a unified way. The power or the energy flow is represented by a half arrow, which is called the power bond or the energy bond $[8,9]$. The causality for each bond is a significant issue that is inherently addressed in bond graph modelling. As every bond involves two power variables, the decision on setting the cause variable and the effect variable is by natural laws. This has a significant bearing on the resulting state equations of the system. Proper assignment of power direction resolves the sign-placing problem when connecting sub-model structures. The causality will dictate whether a specific power variable is a cause or the effect. Using causal bars on either end of the power bond, graphically indicate the causality for every bond. Once the causality is assigned, the bond graph displays the structure of the state space equations explicitly. Examples of engineering systems using bond graph modelling elements are discussed in $[8,9]$.

\subsection{Switching systems in bond graphs: an overview}

Modelling and simulation of switching systems using a bond graph is one of the topics of research. Various models have been proposed. One model uses the ideal switch element [10]. In this model, discontinuous change is handled by enforcing either zero flow $(\mathrm{OFF})$ or zero effort $(\mathrm{ON})$ on 
a junction. However, this approach The shows the bond graph of only one structural mode at any given instant. Also it is unnatural to consider switches as bond graph elements as, unlike other bond graph elements, they do not have energy-related functions. Another approach [11] proposes a switched system description using several bond graph models that depend on switch positions, where the power switch is not considered as a model element. However, for converters containing more switches, this method is cumbersome since the number of combinations is $2^{n}$ where $n$ is the number of switches. Petri net, or finite state machine, is one of the methods to determine the transitions across the modes. This approach is used in [12] for averaged modelling of switching converters. However all relevant discrete system modes and the transition between them have to be explicitly determined before modelling. For $n$ switches in the system, there are $2^{n}$ potential system modes in which all combinations are not physically realisable. In addition, as the switching between the systems is not represented at the energy level, this approach lacks the causality insight that bond graph inherently provides. Use of a modulated transformer MTF to model switching in switching converters is proposed in [13]. It first finds different bond graph models depending on the number of switch positions and then connects these models using MTF elements at appropriate places. However, as the number of switches increases, the model becomes complex. A controlled junction concept has been proposed $[14,15]$. In the ON state, the controlled junction behaves like a normal 0- or 1junction. In the OFF state the 0 -controlled junction forces the effort value at all connected bonds to become 0 . Similarly The 1-controlled junction forces the flow value at all connected bonds to become 0 . This implies that there is no energy transfer across this junction. The control logic for determining the ON and the OFF state for a controlled junction is implemented as a finite state automata and represented as a state transition graph or table. Here the actual switch mechanism is not modelled as a bond graph element. However, changes in configuration across the various operating modes of the system results in reassignment of causality. In [16-18], the switch is modelled as current and voltage source according to the switch state. The OFF condition is modelled as a small current source (leakage current) and the $\mathrm{ON}$ condition is modelled as a small voltage source (forward voltage drop). There is a causality change associated with this change of state of the switch. The 'causality resistors' are introduced to "take the causality change'. Appropriate rules are defined to fix the range of the value of causality resistors. A causality switching algorithm is proposed for swapping of bond variables in the junction structure to represent change in causality and change in the state of the switch. The concept of a junction structure matrix is used to implement this algorithm. The suitability of the algorithm for forced and naturally commutated converters is also presented with examples and computer simulations. The user can model the switching systems without knowing the functional aspects of the switching circuit.

This paper proposes the modelling of switching converters using the concept of switched power junctions [7] where the causal structure remains unchanged even on switching. This type of structure is especially useful for deriving state equations.

\subsection{Concept of switched power junction}

Switched power junction is a generalisation of the junction elements in bond graph, [7]. There are two junction elements in the bond graph. the 0-junction and the 1- junction. The 0-junction represents Kirchoff's current law (KCL) and the 1-junction represents Kirchoff's voltage law (KVL) in the electrical domain. Equations and causal assignments for the 0 and 1 -junction are discussed in the Appendix (Section 8).

At the junctions, there is no dissipation or storage of the power. Incoming power is equal to outgoing power at the junction. If there is an instantaneous discontinuous change in power through the junction instantaneously, then it represents power switching at the junction. This can be achieved by switching flow at the 1 -junction and effort at the 0 -junction. At the 0 -junction only one bond decides the effort. If there are two effort-deciding bonds at the 0 junction and the effort of these bonds is active at the 0junction only at mutually exclusive instants of time then it represents the effort switching at the 0 -junction. Such a 0 junction, that inherently incorporates temporally mutually exclusive multiple effort deciding bonds, is denoted as a 0sjunction. Similarly, a 1-junction where temporally mutually exclusive multiple flow deciding bonds are handled is denoted as a 1s-junction. A 0s-junction is shown in Fig. $1 a$ in which there are two effort-deciding bonds and a 1sjunction is shown in Fig. $1 b$ with two flow-deciding bonds. However, 0s/1s-junctions can be generalised to any number of effort/flow decider bonds.

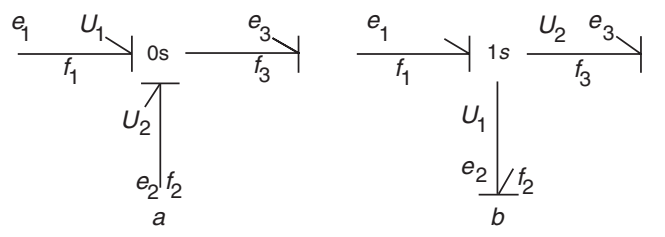

Fig. 1 Switched Power Junctions $a$ 0s-Junction $b$ 1s-Junction

The equations of effort and flow at 0s- and 1s-junctions are:

For 0s-junction

$$
\begin{aligned}
e_{3} & =\text { junction effort } \\
\text { junction effort } & =U_{1} \times e_{1}+U_{2} \times e_{2} \\
f_{1} & =U_{1} \times f_{3} \\
f_{2} & =U_{2} \times f_{3}
\end{aligned}
$$

For 1s-junction

$$
\begin{aligned}
f_{1} & =\text { junction flow } \\
\text { junction flow } & =U_{1} \times f_{2}+U_{2} \times f_{3} \\
e_{2} & =U_{1} \times e_{1} \\
e_{3} & =U_{2} \times e_{1}
\end{aligned}
$$

Effort is denoted as $e$ and flow is denoted as $f$.

$U_{1}$ and $U_{2}$ are the control signals used to switch the junction effort or flow. Boolean algebra governs the multiplication and addition of $U_{1}$ and $U_{2}$. The control signal to switch the junction variable is derived from the control logic, which is specific to that system. $U_{1}$ and $U_{2}$ are placed at the corresponding effort or flow decider bonds, whose effort or flow is active when either $U_{1}$ is 1 or $U_{2}$ is 1 . The example considered two effort/flow deciding bonds. Figure 1 is given for two effort/flow decider bonds, however, $0 \mathrm{~s} / 1 \mathrm{~s}$ junctions can be generalised to any number of effort/flow decider bonds. 


\subsection{Switched power junctions and power electronic systems}

Power semiconductor switches such as diode, MOSFET, power transistor etc. are an integral part of switching converters in power electronic systems. All power semiconductor switches are basically a single pole single throw (SPST) type of switch. To analyse the functional performance of converters, the semiconductor switches can be considered as ideal SPST switches. The state of the switch can be controlled by generating signals that depend on the converter topology and the properties of the semiconductor device. By using the SPST switch as the basic unit, one can realise all switching circuits in power electronic systems.

The SPST switch can be modelled as a 1s-junction with two flow decider bonds as shown in Fig. 2. $U$ and $\bar{U}$ are control signals that control the junction flows. When the switch is closed, i.e. when $U=1$ junction flow is determined by the system. When the switch is open, i.e. when $\bar{U}=1$, flow at as the junction is zero. Therefore one flow decider bond is modelled as the zero current source and the other flow decider bond is connected to the system. Thus the function of the switch is emulated.

Combinations of SPST switches can be modelled using 0s-and 1s-junctions. For example, the H-bridge as shown in Fig. $3 a$ is shown as combination of SPST switches. The circuit in Fig. $3 a$ is a single-phase inverter using a controlled switch such as a MOSFET. However, the H-bridge can be a diode bridge rectifier with source and load position interchanged which is not considered in this example. All these switches can be modelled as SPST switches. The bond graph of the H-bridge is shown in Fig. $3 b$. Individual SPST switch function is modelled as 1s-junction and the points $\mathrm{O}_{1}$ and $\mathrm{O}_{2}$ are modelled as 0s-junctions since there is effort switching. The purpose of this example is to show the generality of application of switched power junctions to power electronic systems. It is important to note that the user should know the functional aspects of the circuit for using switched power junctions for modelling. This concept is now applied to model PWM DC-DC power converters.

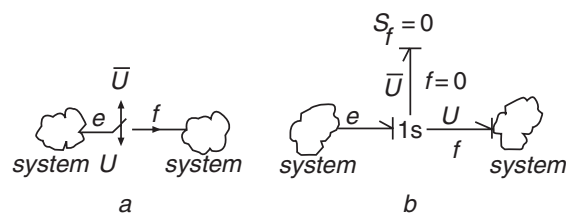

Fig. 2 SPST Switch

$a$ SPST switch

$b$ bond graph model of SPST switch

\section{$3 \quad$ Modelling methodology}

The switched model power converter circuit contains switches and supposedly linear circuit elements. Switches are responsible for the nonlinear behaviour of the converters. In most converters, there is a combination of switches in the form of a single pole double throw (SPDT) switch. The SPDT switch is a combination of two SPST switches. In most converter circuits combinations of one active switch (transistor or MOSFET) and one passive switch (diode) realise the SPDT switch. Figure 4 shows an SPDT switch, its SPST realisation and two combinations of MOSFET and diode, which realise the SPDT switch. The bond graph model for this basic switching block is the object of focus here.

A single switch is modelled as a SPST switch. Using switched power junctions, switch network is modelled as a bond graph as shown in Fig. 5. The SPST switch is modelled as a 1s-junction and the node $\mathrm{O}$ is modelled as a 0s-junction. Here continuous conduction mode (CCM) is considered. Discontinuous conduction mode (DCM) operation is discussed in Section 5. Switches $S_{1}$ and $S_{2}$ are closing mutually exclusive in time with respect to each other. The control signals $U$ and $\bar{U}$ (instead of $U_{1}$ and $U_{2}$ ) activate the switches $S_{1}$ and $S_{2}$, respectively, i.e. when $U=1$, $\mathrm{S}_{1}$ is $\mathrm{ON}$ and when $\bar{U}=1, \mathrm{~S}_{2}$ is $\mathrm{ON}$. $U$ and $\bar{U}$ are the Boolean signals and are complement of each other such that $U+\bar{U}=1$. These signals are derived from a control logic, which is derived according to the switch property. For example, if $\mathrm{S}_{1}$ is a MOSFET, $U$ is given from the duty cycle controller and if $S_{2}$ is a diode, $\bar{U}$ is derived from the duty cycle controller as well as the current in $S_{2}$. In the bond graph, one can incorporate these signals in switched power
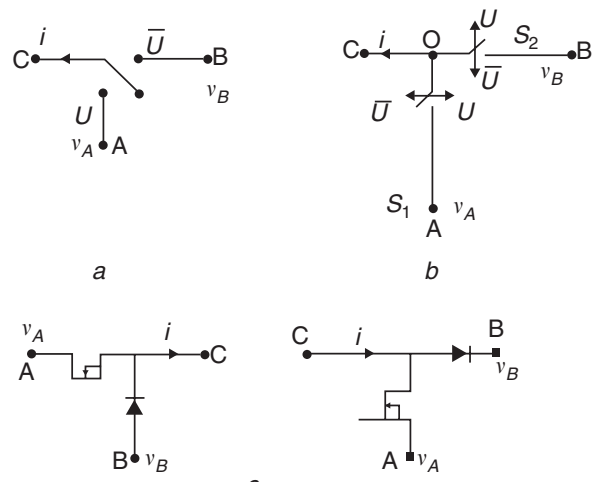

Fig. 4 SPDT switch

$a$ SPDT switch

$b$ SPST switch realization of SPDT switch

$c$ examples of SPDT switch realization using circuit elements

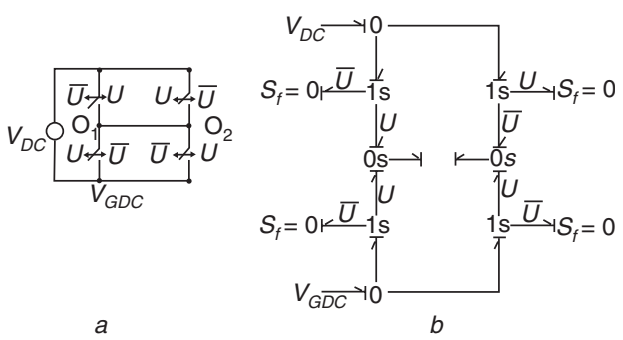

Fig. 3 H-bridge inventor. $a$ H-Bridge single phase inverter $b$ bond graph model of $\mathrm{H}$-bridge inverter

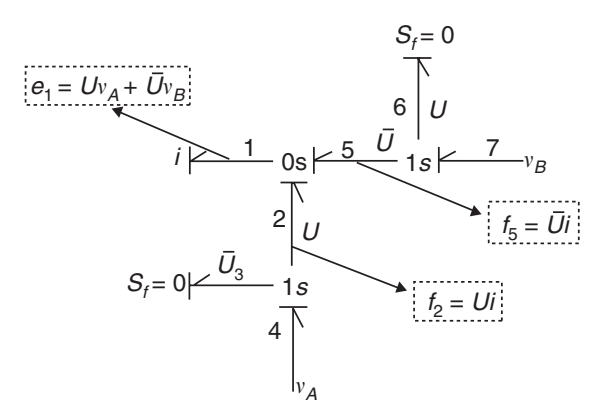

Fig. 5 Bond graph realisation of SPDT switch 
junctions as in Fig. 5. It should be noted that the bonds 2 and 5 in Fig. 5 are not switching bonds. The presence of $U$ and $\bar{U}$ is merely a notational convenience. It means that the junction effort and/or flow is taken from these mutually exclusive bonds at $U$ and $\bar{U}$ times.

The main objective here is to establish the averaged large signal, steady-state and small signal models by graphical reduction. This would ultimately enable one to obtain the large and small signal state equations by inspection. The bond graph model of the switch shown in Fig. 5 describes the large signal behaviour of the switch network. To obtain the averaged large signal model of the switch network, the following graphical steps are applied.

Step 1 Inspect the bonds that have zero power. In Fig. 5, the zero power bonds are 3 and 6 since flow in them is zero. Remove these bonds from the graph. The resulting graph is shown in Fig. $6 a$.

Step 2 Referring to Fig. $6 a$, merge bonds 2 and 4 and also merge bonds 5 and 7 as the 1s-junctions have less than three bonds connected to them. The resulting graph is shown in Fig. $6 b$.

Referring to Fig. $6 b$, the causality on bonds 2,4 and 5,7 indicates that the bond is effort causal at the 0s-junction. The Boolean terms will appear as a multiplier in the 'effect' variables, which in this case is the flow. The flow at bond 1 is the effect from the external circuit but the effort is decided either by bond 2,4 or bond 5,7 depending on the Boolean signals. This dependency is seen in the effort equation of bond 1 as indicated in Fig. $6 b$.

Step 3 For CCM operation, $U$ and $\bar{U}$ are each other complement and are rectangular pulse trains of duty cycle $d$ and 1- $d$, respectively. To obtain the averaged model, replace $U$ and $\bar{U}$ in Fig. $6 b$ by $d$ and 1- $d$ where $d$ and 1- $d$ are time averaged values of $U$ and $\bar{U}$. The resulting graph that is shown in Fig. $7 a$ is the averaged large signal bond graph model of the switch network.

Step 4 To obtain the steady-state model, in Fig. $7 a$ replace $d$ by $D, e$ by $E$ and $f$ by $F$, where $D, E$ and $F$ are the steadystate operating points of the duty cycle, the effort and the

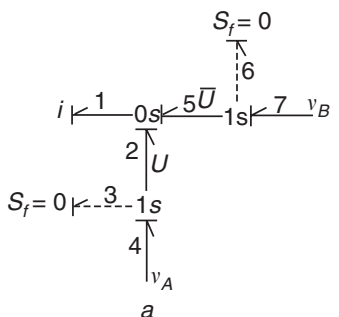

a

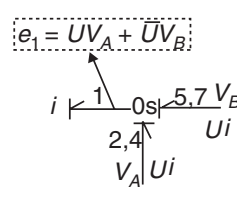

$b$
Fig. 6 SPDT switch bond graph

$a$ removal of zero power bonds from SPDT switch bond graph $b$ bond graph of SPDT switch after merging two bonds

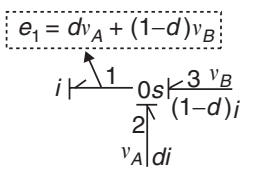

a

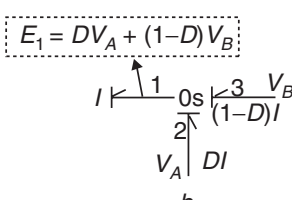

b
Fig. 7 Switch network $a$ averaged large signal model $b$ steady-state model flow, respectively. The switch network in steady state is shown in Fig. $7 b$.

Step 5 To obtain the small signal AC model, the principle of applying a small perturbation in the neighbourhood of the steady-state operating point is used graphically.

Introduce small perturbations [2] around the operating point in $d$, $e$ and $f$ of each bond as

$$
\begin{aligned}
& d=D+\hat{d} \\
& e=E+\hat{e} \\
& f=F+\hat{f}
\end{aligned}
$$

where $\hat{d}, \hat{e}$ and $\hat{f}$ are small perturbations in duty cycle $d$, effort $e$ and flow $f$ around operating point $D, E$ and $F$, respectively.

Step 6 Referring to Fig. $7 a$, the product of two quantities with perturbation like $d^{*} e$ and $d^{*} f$ can be written as

$$
\begin{gathered}
d * e=(D+\hat{d}) *(E+\hat{e})=D E+D \hat{e}+E \hat{d}+\hat{e} \hat{d} \\
d * f=(D+\hat{d}) *(F+\hat{f})=D F+D \hat{f}+F \hat{d}+\hat{f} \hat{d}
\end{gathered}
$$

By neglecting second-order perturbations $\hat{e} \hat{d}, \hat{f} \hat{d}$ and by removing DC terms $D E$ and DF from (3) and (4), the firstorder small signal AC terms are obtained. Referring to Fig. $7 a$, the bond 1 effort equation is given by

$$
\hat{e}_{1}=D \hat{v}_{A}+V_{A} \hat{d}+(1-D) \hat{v}_{B}-V_{B} \hat{d}
$$

It is evident from (5) that new perturbation terms are added to the effort equation at bond 1 due to the product of two quantities that includes the perturbation effect. The new terms can be equivalently represented as input effort sources due to perturbation in duty cycle as indicated in Fig. 8.

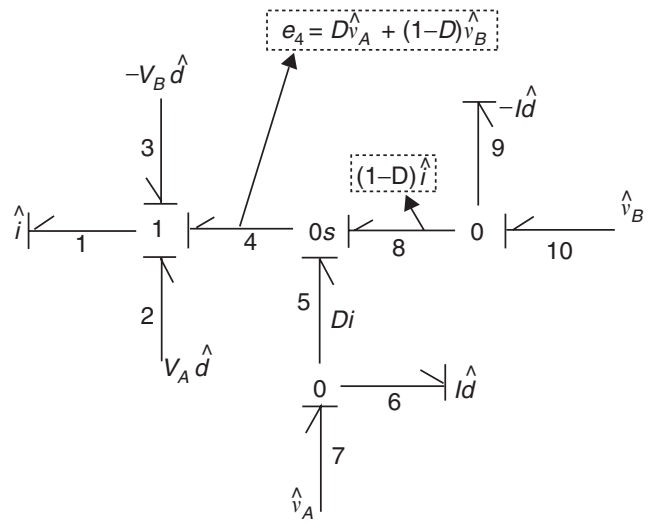

Fig. 8 Small signal ac model of the switch network

Referring to Fig $7 a$, it can be seen that the bond 2 flow equation is given by

$$
\hat{f}_{1}=D \hat{i}+I \hat{d}
$$

From (6) the new perturbation terms that are added to the flow equation at bond 2 can be equivalently represented as input flow sources due to the perturbation in the duty cycle as indicated in Fig. 8.

Referring to Fig. $7 a$, the bond 3 flow equation is given by

$$
\hat{f}_{2}=D \hat{i}-I \hat{d}
$$

From (7) the new perturbation terms that are added to the flow equation at bond 3 can be equivalently represented as input sources due to the perturbation in the duty cycle as indicated in Fig. 8. Figure 8 now gives the small signal AC model of the switch network. 
In some converters, combination of switches as shown in Fig. 9 occurs (e.g. Cuk and SEPIC) frequently. The bond graph for this combination is shown in Fig. 10, where the additional 0s-junction is formed by bonds 12-14. When $\mathrm{S}_{1}$ is $\mathrm{ON}, v$ is connected to the ground with respect to point $\mathrm{O}_{1}$. When $\mathrm{S}_{2}$ is $\mathrm{ON} v$ is connected to ground with respect to point $\mathrm{O}_{2}$. This is to provide the reference ground to the system modelled using the bond graph. Once the model is formed one can apply a graphical reduction algorithm to it. Applying steps 1-3 discussed above, one obtains the averaged large signal bond graph model as shown in Fig. 11. It should may be noted that one can remove bonds 12-14 in the second step since its significance for obtaining the averaged large signal, steady-state and small signal modelling is null.

The small signal AC model is also obtained along similar lines, as discussed previously from steps 4-6. The small signal model in this case is shown in Fig. 12.

As mentioned earlier, these switch networks are the basic building blocks in SMPCs. In general, one has to trace the points where effort and flow switches. By placing the appropriate switching power junction, the bond graph model of the converter can be obtained which is large signal model. If there is switching in the reference ground as shown in Fig. 9, then one has to put the additional junction to provide the appropriate reference ground according to switching state as in Fig. 10. Once the large signal bond graph model is obtained, the averaged large signal, steadystate and small signal models of the SMPCs can be obtained by using the graphical reduction process illustrated in this Section. Using the large signal and the small signal bond graph models, the state equations representing the SMPCs dynamics can be obtained directly by inspection.

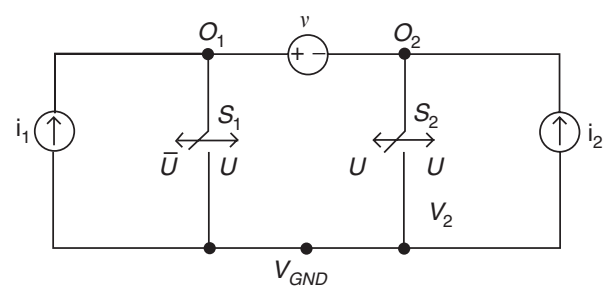

Fig. 9 Commonly occurring switch network

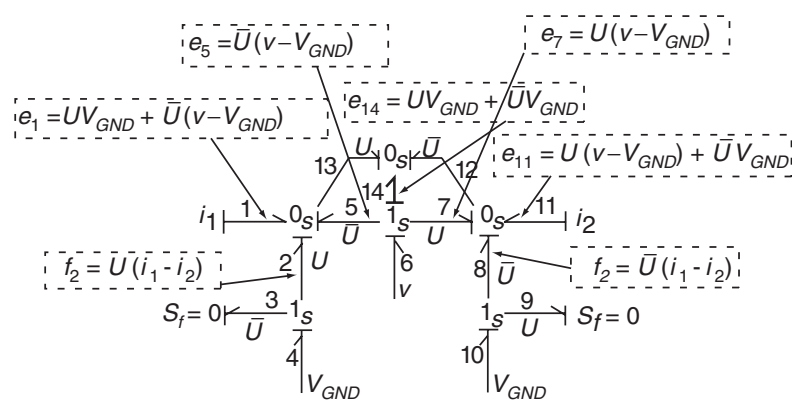

Fig. 10 Bond graph of the switch network in Fig. 9

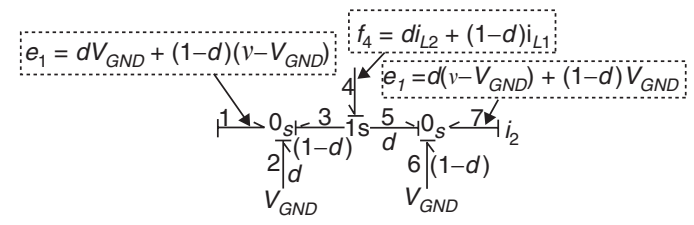

Fig. 11 Averaged large signal bond graph model of the switch network of Fig. 9

\section{Modelling of switching converters in CCM}

This Section discusses the modelling of switching converters in CCM. Two basic converters namely boost and Cuk are taken as examples. Figure 13 shows a boost converter. Switches $\mathrm{S}_{1}$ and $\mathrm{S}_{2}$ are connected at point $\mathrm{O}$, which forms the SPDT switch as described in section 3. $\mathrm{S}_{1}$ represents the MOSFET and $\mathrm{S}_{2}$ represents the diode connected as shown in Fig. 13. Figure 14 shows a boost converter large signal bond graph model. There are two state variables in the converter, $i_{L}$ and $v_{O}$. Referring to the bond graph of Fig. 14, it is seen that the flow of bond 2 is the $i_{L}$ state variable and effort at bond 11 is the other state variable, $v_{O}$. Therefore bond 2 is the flow decider for the corresponding 1-junction and effort at bond 2 gives the first state equation, which is given by $\mathrm{KVL}$ at the corresponding 1-junction as

$$
e_{2}=L \frac{d i_{L}}{d t}=v_{g}-i_{L} R_{L}-\bar{U} v_{O}
$$

Similarly bond 11 is the effort decider for the corresponding 0 -junction. Therefore flow at bond 11 is the second state equation, which is given by KCL at the corresponding 0junction as

$$
f_{11}=C \frac{d v_{O}}{d t}=\bar{U} i_{L}-\frac{v_{O}}{R}
$$

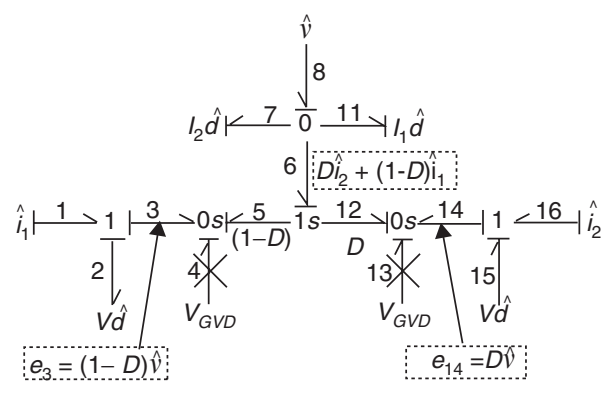

Fig. 12 Small signal AC bond graph model of the switch network of Fig. 9

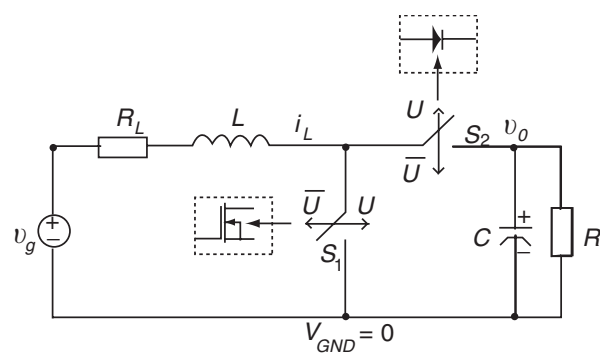

Fig. 13 Boost converter

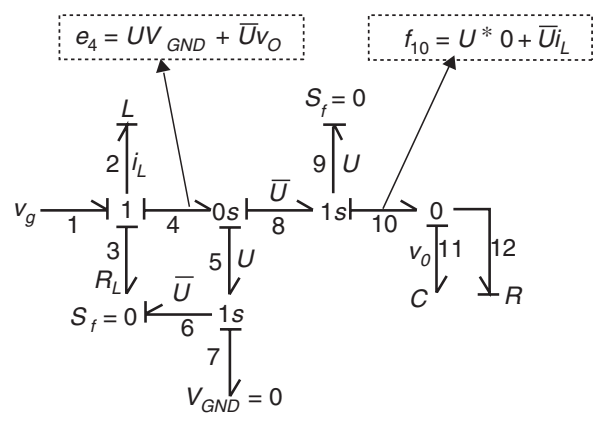

Fig. 14 Bond graph model of the boost converter 
As discussed in Section 3, we apply the steps for graphical reduction to obtain the averaged large signal, steady-state and the small signal graphs. Applying step 1, the zero power bonds 6 and 9 are removed from the bond graph. According to step 2 bonds 5,7 and 8,10 are merged. The resulting graph is shown in Fig. 15a. After executing step 3, the averaged large signal model is given in Fig. 15b. The state equation for the large signal averaged model shown in Fig. $15 b$ is given by

$$
\begin{aligned}
{\left[\begin{array}{cc}
L & 0 \\
0 & C
\end{array}\right] \frac{d}{d t}\left[\begin{array}{c}
i_{L} \\
v_{O}
\end{array}\right]=} & {\left[\begin{array}{cc}
-R_{L} & -(1-d) \\
(1-d) & -1 / R
\end{array}\right]\left[\begin{array}{c}
i_{L} \\
v_{O}
\end{array}\right] } \\
& +\left[\begin{array}{l}
1 \\
0
\end{array}\right] v_{g}
\end{aligned}
$$

After executing step 4, one obtains the steady-state model of the converter. This model is useful to calculate the performance indices such as efficiency and conversion ratio. In the steady state, the derivatives of states $i_{L}$ and $v_{O}$ are zero since these terms are DC. Therefore the LHS of (10) is zero. Equating the terms of the LHS to zero and replacing all the variables by their DC values, one obtain the steadystate equations as

$$
\begin{aligned}
& -I_{L} R_{L}-(1-D) V_{O}+V_{g}=0 \\
& (1-D) I_{L}-\frac{V_{O}}{R}=0
\end{aligned}
$$

For instance, e.g. one can obtain the voltage conversion ratio from (11) as

$$
\frac{V_{O}}{V_{g}}=\frac{(1-D)}{\frac{R_{L}}{R}+(1-D)^{2}}
$$

Now, to obtain the small signal AC model, execute step 5 and step 6 . The small signal ac bond graph model is given in Fig. 17. The small signal AC state space equation is given as

$$
\begin{aligned}
{\left[\begin{array}{ll}
L & 0 \\
0 & C
\end{array}\right] \frac{d}{d t}\left[\begin{array}{c}
\hat{i}_{L} \\
\hat{v}_{O}
\end{array}\right]=} & {\left[\begin{array}{cc}
-R_{L} & -(1-D) \\
(1-D) & -\frac{1}{R}
\end{array}\right]\left[\begin{array}{c}
\hat{i}_{L} \\
\hat{v}_{O}
\end{array}\right] } \\
& +\left[\begin{array}{l}
1 \\
0
\end{array}\right] \hat{v}_{g}+\left[\begin{array}{c}
V_{O} \\
-I_{L}
\end{array}\right] \hat{d}
\end{aligned}
$$
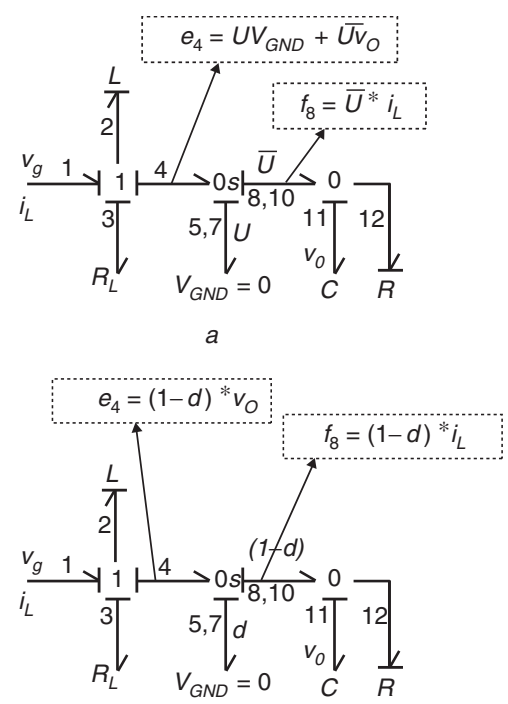

$b$

Fig. 15 Boost conveter $a$ Reduced bond graph $b$ Averaged large signal model
There is a cross on bond 6 , which indicates that bond 6 is the zero power bond in this case and hence is neglected for obtain the small signal model.

In this way the large signal, steady-state and small signal AC models of the boost converter are obtained graphically. It is clear from the procedure that in a few steps, starting from the large signal model bond graph, the steady state and small signal AC models are obtained. When one is familiar with the graphical procedure one can obtain these models in just three steps.

The example of the Cuk converter in CCM given below consolidates this approach. The Cuk converter is given in Fig. 18, and its bond graph model is shown in Fig. 19. This switch network is same as that described in Fig. 9.

After executing steps $1-3$, one obtains the large signal averaged model as shown in Fig. 20. The bonds 18-20 are

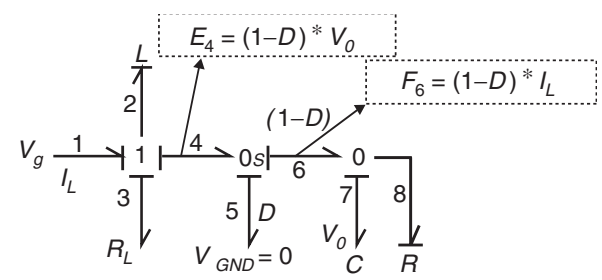

Fig. 16 Steady state bond graph model of boost converter

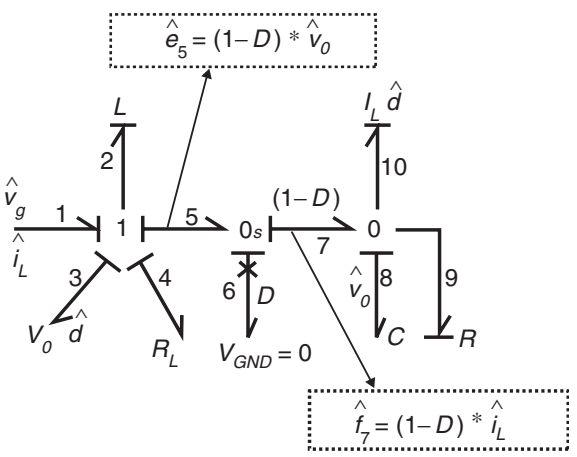

Fig. 17 Small signal ac bond graph model of boost converter

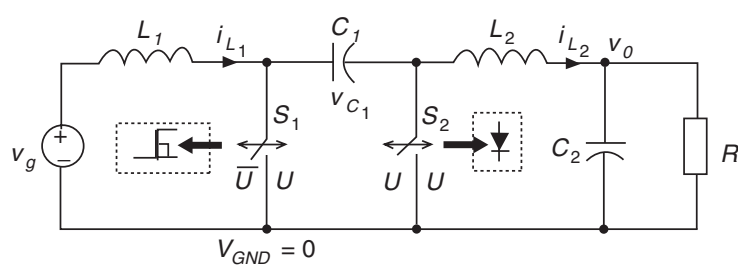

Fig. 18 Cuk converter

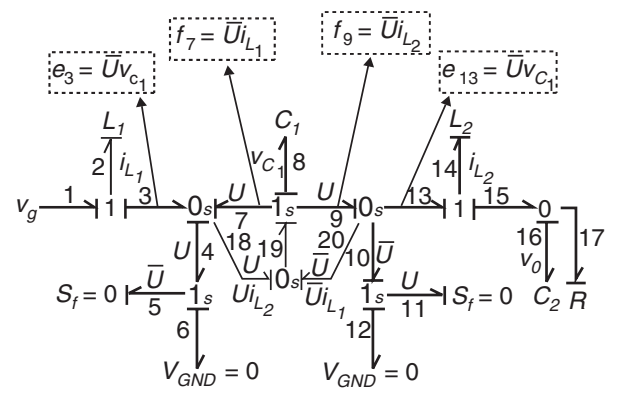

Fig. 19 Bond graph model of Cuk converter 
removed since they are zero power bonds as well as having null significance in obtaining the averaged model.

$$
\begin{aligned}
& {\left[\begin{array}{cccc}
L_{1} & 0 & 0 & 0 \\
0 & L_{2} & 0 & 0 \\
0 & 0 & C_{1} & 0 \\
0 & 0 & 0 & C_{2}
\end{array}\right] \frac{d}{d t}\left[\begin{array}{c}
i_{L_{1}} \\
i_{L_{2}} \\
v_{C_{1}} \\
v_{0}
\end{array}\right]} \\
& =\left[\begin{array}{cccc}
0 & 0 & -\bar{U} & 0 \\
0 & 0 & -U & -1 \\
\bar{U} & U & 0 & 0 \\
0 & 1 & 0 & -\frac{1}{R}
\end{array}\right]\left[\begin{array}{c}
i_{L_{1}} \\
i_{L_{2}} \\
v_{C_{1}} \\
v_{0}
\end{array}\right]+\left[\begin{array}{l}
1 \\
0 \\
0 \\
0
\end{array}\right] v_{g}
\end{aligned}
$$

Equation (14) is the state equation of the large signal model of the Cuk converter.

$$
\begin{aligned}
& {\left[\begin{array}{cccc}
L_{1} & 0 & 0 & 0 \\
0 & L_{2} & 0 & 0 \\
0 & 0 & C_{1} & 0 \\
0 & 0 & 0 & C_{2}
\end{array}\right] \frac{d}{d t}\left[\begin{array}{c}
i_{L_{1}} \\
i_{L_{2}} \\
v_{C_{1}} \\
v_{0}
\end{array}\right]} \\
& =\left[\begin{array}{cccc}
0 & 0 & -(1-d) & 0 \\
0 & 0 & -d & -1 \\
(1-d) & d & 0 & 0 \\
0 & 1 & 0 & -\frac{1}{R}
\end{array}\right]\left[\begin{array}{c}
i_{L_{1}} \\
i_{L_{2}} \\
v_{C_{1}} \\
v_{0}
\end{array}\right] \\
& +\left[\begin{array}{c}
1 \\
0 \\
0 \\
0
\end{array}\right] v_{g}
\end{aligned}
$$

Step 4 gives the steady-state model. After executing step 5 and 6 one obtains the small signal AC model of the Cuk converter as shown in Fig. 21. The state equations for the small signal AC model are obtained from the small signal graph of Fig. 21 as

$$
\begin{aligned}
& {\left[\begin{array}{cccc}
L_{1} & 0 & 0 & 0 \\
0 & L_{2} & 0 & 0 \\
0 & 0 & C_{1} & 0 \\
0 & 0 & 0 & C_{2}
\end{array}\right] \frac{d}{d t}\left[\begin{array}{c}
\hat{i}_{L_{1}} \\
\hat{i}_{L_{2}} \\
\hat{v}_{C_{1}} \\
\hat{v}_{0}
\end{array}\right]} \\
& =\left[\begin{array}{cccc}
0 & 0 & -(1-D) & 0 \\
0 & 0 & -D & -1 \\
(1-D) & D & 0 & 0 \\
0 & 1 & 0 & -\frac{1}{R}
\end{array}\right]\left[\begin{array}{c}
\hat{i}_{L_{1}} \\
\hat{i}_{L_{2}} \\
\hat{v}_{C_{1}} \\
\hat{v}_{0}
\end{array}\right] \\
& +\left[\begin{array}{l}
1 \\
0 \\
0 \\
0
\end{array}\right] \hat{v}_{g}+\left[\begin{array}{c}
V_{C_{1}} \\
-V_{C_{1}} \\
-I_{L_{1}}+I_{L_{2}} \\
0
\end{array}\right] \hat{d}
\end{aligned}
$$

The modelling procedure for SMPCs in CCM is illustrated through the boost and Cuk converter examples. A switched power converter of any complexity can be modelled using the above procedure.

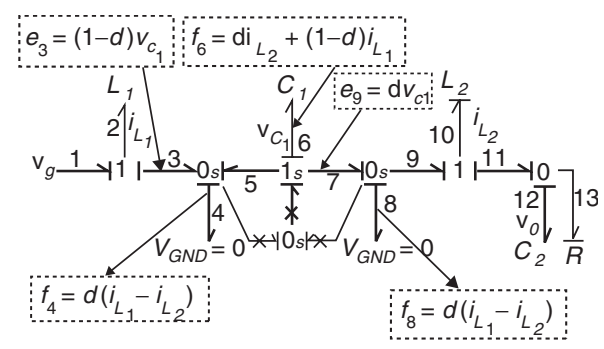

Fig. 20 Averaged large signal bond graph model of Cuk converter

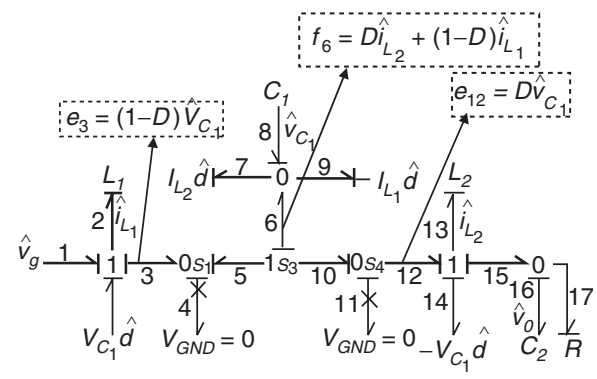

Fig. 21 Small signal ac bond graph model of Cuk converter

\section{Modelling of switching converters in DCM}

The graphical modelling procedure of PWM DC-DC power converters in DCM is discussed in this Section. The discontinuous inductor current mode (DICM) is discussed here. When the PWM converter operates in DICM, the inductor current reaches zero before one switching cycle is over. Conventionally this phenomenon is modelled using a reduced-order model of the converter. The problems with state-order reduction models are discussed in $[4,19,20]$. To prevent the order reduction in the converter model, a fictitious device called the discontinuous mode switching device (DMSD), which is a fourterminal device has been proposed [19]. Its main function lies in the topological support to the formulation of the fullorder state space model. Along similar lines, this paper introduces the virtual switch to prevent the order reduction in the state space model. As an example, the virtual switch
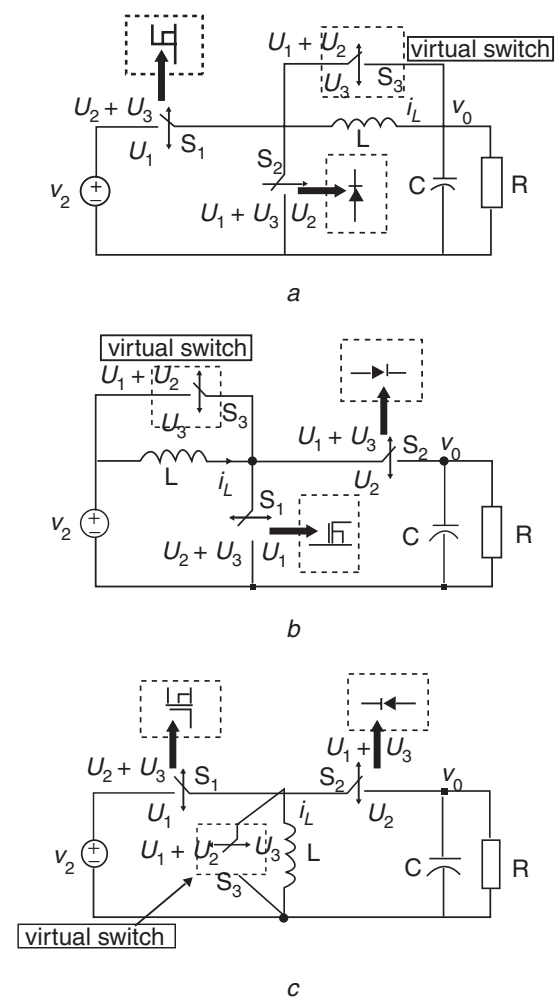

Fig. 22 Equivalent circuits of basic converters in DCM by using virtual switching $a$ buck converter $b$ boost converter $c$ buck-boost converter 
position in three basic converters is shown in Fig. 22. A buck converter example is chosen for modelling (Fig. 22a). In DCM when the inductor current reaches zero, both the controlled switch $\mathrm{S}_{1}$ and diode $\mathrm{S}_{2}$ obtains OFF. The voltage at point $\mathrm{O}$ becomes $v_{O}$. This virtual switch only closes when both switches become OFF. $U_{1}, U_{2}$ and $U_{3}$ are the Boolean signals, which are mutually exclusive in time with respect to each other. These Boolean signals control the states of the switches $S_{1}, S_{2}$ and $S_{3}$ shown in Fig. $22 a$. The bond graph model of converter in Fig. $22 a$ is as shown in Fig. 23. Fig. 23 is the large signal model of the converter in DCM. State equations for the large signal model are given as

$$
\begin{aligned}
{\left[\begin{array}{ll}
L & 0 \\
0 & C
\end{array}\right] \frac{d}{d t}\left[\begin{array}{c}
i_{L} \\
v_{O}
\end{array}\right]=} & {\left[\begin{array}{cc}
0 & -\left(U_{1}+U_{2}\right) \\
\left(U_{1}+U_{2}\right) & -\frac{1}{R}
\end{array}\right]\left[\begin{array}{c}
i_{L} \\
v_{O}
\end{array}\right] } \\
& +\left[\begin{array}{c}
U_{1} \\
0
\end{array}\right] v_{g}
\end{aligned}
$$

The rest of the procedure to obtain the large signal averaged model, steady-state and small signal AC model is the same as was described for the CCM in Section 4. The only change will be that, instead of $U$ and $\bar{U}$, there will be three control signals $U_{1}, U_{2}$ and $U_{3}$. The sum of $U_{1}, U_{2}$ and $U_{3}$ i.e. $U_{1}+U_{2}+U_{3}=1$. The time average of $U_{1}, U_{2}$ and $U_{3}$ are $d_{1}, d_{2}$ and $\left(1-d_{1}-d_{2}\right)$. The duty cycle controller gives $d_{1} . d_{2}$ is a function of the parameters of the circuit, switching frequency as well as $d_{1} \cdot d_{3}$ is a function of $d_{1}$ and $d_{2}$. After executing steps $1-3$ one obtains the large signal averaged model as shown in Fig. 24a. The state equations are

$$
\begin{aligned}
{\left[\begin{array}{cc}
L & 0 \\
0 & C
\end{array}\right] \frac{d}{d t}\left[\begin{array}{c}
i_{L} \\
v_{O}
\end{array}\right]=} & {\left[\begin{array}{cc}
0 & -\left(d_{1}+d_{2}\right) \\
\left(d_{1}+d_{2}\right) & -\frac{1}{R}
\end{array}\right]\left[\begin{array}{c}
i_{L} \\
v_{O}
\end{array}\right] } \\
& +\left[\begin{array}{c}
d_{1} \\
0
\end{array}\right] v_{g}
\end{aligned}
$$

To obtain the get steady-state model, execute step 4 . Figure $24 b$ shows the steady-state graph. The equations obtained from this graph give the steady-state performance indices.

$$
\begin{aligned}
& D_{1} V_{g}-\left(D_{1}+D_{2}\right) V_{O}=0 \\
& \left(D_{1}+D_{2}\right) I_{L}-\frac{V_{O}}{R}=0
\end{aligned}
$$

The voltage conversion ratio obtained from (19) is

$$
\frac{V_{O}}{V_{g}}=\frac{D_{1}}{D_{1}+D_{2}}
$$

To obtain the small signal AC model, execute steps 5 and 6 .

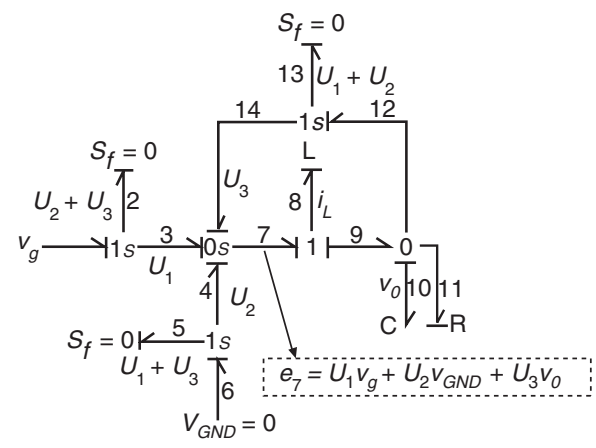

Fig. 23 Bond graph model of buck converter in DCM
The small signal AC bond graph model is shown in Fig. 25. The small signal AC state space equations are

$$
\begin{aligned}
{\left[\begin{array}{ll}
L & 0 \\
0 & C
\end{array}\right] \frac{d}{d t}\left[\begin{array}{c}
\hat{i}_{L} \\
\hat{v}_{O}
\end{array}\right]=} & {\left[\begin{array}{cc}
0 & -\left(D_{1}+D_{2}\right) \\
\left(D_{1}+D_{2}\right) & -\frac{1}{R}
\end{array}\right]\left[\begin{array}{c}
\hat{i}_{L} \\
\hat{v}_{O}
\end{array}\right] } \\
& +\left[\begin{array}{c}
D_{1} \\
0
\end{array}\right] \hat{v}_{g}+\left[\begin{array}{c}
V_{g}-V_{0} \\
-I_{L}
\end{array}\right] \hat{d}_{1} \\
& +\left[\begin{array}{c}
-V_{0} \\
-I_{L}
\end{array}\right] \hat{d}_{2}
\end{aligned}
$$

Note the appearance of the term $\hat{d}_{2}$, it is considered as a control input. and can be expressed in terms of $\hat{d}_{1}$ and other variables [20].

Therefore using the virtual switch concept, switched power converters in DCM can also be modelled using the bond graphical approach without reducing the state order of the converter model. For calculation of $d_{2}$, however, one has to use analytical approach described in $[20]$.
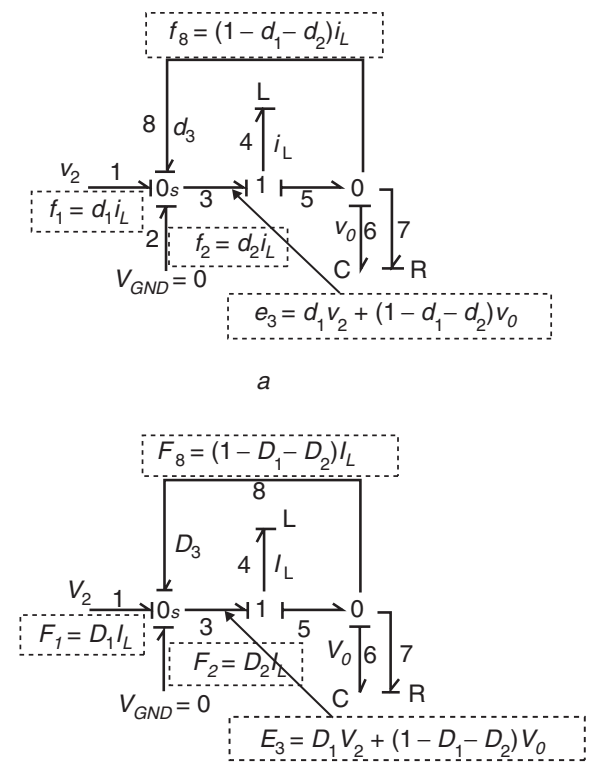

Fig. 24 Bond graph model

$a$ averaged large signal bond graph model $b$ Steady state bond graph model

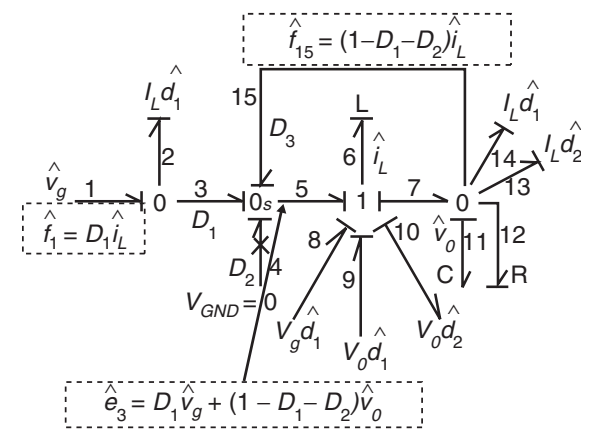

Fig. 25 Small signal AC bond graph model of buck converter in $D C M$ 


\section{Conclusions}

The bond graph approach to model switched mode power converters has been presented in the paper. The Switching phenomenon is modelled using the concept of switched power junctions. Bond graph modelling of the switched power converter is described to obtain the large signal averaged, steady-state and small signal AC model of the switched power converters.

The bond graph of the converter is the large signal model of the converter. A graphical procedure is proposed that gives the averaged large signal, steady-state and small signal AC models. The procedure is suitable for both converters in CCM and in DCM.

Modelling of converters in CCM is illustrated using examples of boost and Cuk converters. For modelling in DCM, the concept of the virtual switch is used to model the converter using the bond graph. The concept of the virtual switch topologically supports the formulation of the fullorder model of the converter in DCM. The DCM case is illustrated using the buck converter.

Using the above procedures, converters of any complexity can be modelled incorporating all the advantages of bond graph modelling. Before using this method, one needs to be familiar with the functionality of the converter. The proposed method enhances the visual understanding of the cause-effect relationship between voltage-current variables in the circuit. The bond graph of the converter displays all the physically realisable switching states in a single graph. Further, the bond graph of the converter explicitly displays the structure of the dynamic equations of the converter.

\section{References}

1 Erickson, R.W., Cuk, S., and Middlebrook, R.D.: 'Large signal modelling and analysis of switching regulators'. Proceedings of IEEE PESC, Cambridge, MA, USA, 1982, pp. 240-250

2 Cuk, S., and Middlebrook, R.D.: 'A general unified approach to modelling switching-converter power stages'. Proceedings IEEE PESC, Cleveland, OH, USA, 1976, pp. 18-34

3 Wester, G.W., and Middlebrook, R.D.: 'Low frequency characterization of switched DC-DC converters', IEEE Trans. Aerosp. Electron. Sys., 1973, 9, pp. 376-385

4 Vorperian, V.: 'Simplified analysis of PWM converters using the model of PWM switch: Parts I and II', IEEE Trans. Aerospace Electron. Syst., 1990, 26, pp. 490-505

5 Van Dijk, E., Spruijt, H.J.N., Sullivan, D.M.O., and Klaassens, J.B.: 'PWM switch modelling of DC-DC converters', IEEE Trans. Power Electron., 1995, 10, (6), pp. 659-665

6 Smedley, K., and Cuk, S.: 'Switching flow-graph nonlinear modelling technique', IEEE Trans. Power Electron., 1994, 9, (4), pp. 405-413

7 Umanand, L.: 'Switched junctions in bond graph for modelling power electronic systems', J. Indian Inst. Sci., 2000, pp. 327-332

8 Karnopp, D.C., and Rosenberg, R.C.: 'System dynamics a unified approach', (Wiley Interscience Publications, USA, 1975)

9 Mukherjee, A., and Karmakar, R.: 'Modelling and simulation of engineering systems through bond graphs', (Narosa Publishing House, India, 2000)

10 Stromberg, J.E., Top, J., and Soderman, U.: 'Variable causality in bond graph caused by discrete effects'. Proceedings of SCS Conference ICBGM'95, San Diego, CA, USA, 1993, pp. $115-119$

11 Demir, Y., and Poyraz, M.: ' Derivation of state and output equations for systems containing switches and a novel definition of switch using the bond graph model', J. Franklin Inst., 1997, 3343, (2), pp. 191-197

12 Allard, B., Helali, H., Lin, C., and Morel, H.: 'Power converter average model computation using the bond graph and petri net techniques'. Proceedings of IEEE PESC, Atlanta, GA, USA, 1995, pp. $830-836$

13 Delgado, M., and Sira-Ramirez, H.: 'A bond graph approach to the modelling and simulation of regulated dc-dc power supplies', Simul. Pract. Theory Vol. 6, 1998, 12, pp. 631-646

14 Mosterman, P.J., and Biswas, G.: 'Behaviour generation using model switching- a hybrid bond graph modelling technique'. International
Conference on Bond graph modelling, Las Vegas, NV, USA, 1995, pp. $177-182$

15 Mosterman, P.J., and Biswas, G.: 'A theory of discontinuities in physical systems model', J. Franklin Inst., 1998, 335B, (3), pp. 401-439

16 Asher, G.M., and Eslamdoost, V.: 'Power electronic circuit simulation and bond graph techniques'. EPE Aachen, Germany, Oct 1989, Vol. 2, pp. 807-812

17 Asher, G.M., and Eslamdoost, V.: 'A novel causality changing method for the bond modelling of variable topology electronic switching circuits'. Proceedings of IMACS Symposium, Lille, Belgium, 1991, pp. 371-377

18 Asher, G.M.: 'The robust modelling technique of variable topology circuits using bond graphs'. Proceedings of ICBGM 93, San Diego, CA, USA, pp. 126-131

19 Femia, N., and Tucci, V.: 'On the modelling of PWM converters for large signal analysis in discontinuous conduction mode', IEEE Trans. Power Electron., 1994, 9, (5), pp. 487-496

20 Sun, J., Mitchell, D.M., Greuel, M.F., Krein, P.T., and Bass, R.M.: 'Averaged modelling of PWM converters operating in discontinuous conduction mode', IEEE Trans. Power Electron., 2001, 16, (4), pp. 482-492

\section{Appendix}

\section{Structure and equations of the 0-junction and 1-junction \\ 8.1 0-Junction.}

Figure 26 shows the 0 -junction. At the 0 -junction, only one bond at the junction decides the effort at the junction and hence the effort at all bonds. The flow at all the bonds except the effort deciding bond decides flow at the effort deciding bond. In Figure 26, bond 1 decides the effort at all bonds and bonds 2-6 decide the flow at bond 1 . In equation form

$$
\begin{aligned}
& e_{2}=e_{1} \\
& e_{3}=e_{1} \\
& e_{4}=e_{1} \\
& e_{5}=e_{1} \\
& e_{6}=e_{1} \\
& f_{1}=f_{2}+f_{3}+f_{4}+f_{5}+f_{6}
\end{aligned}
$$

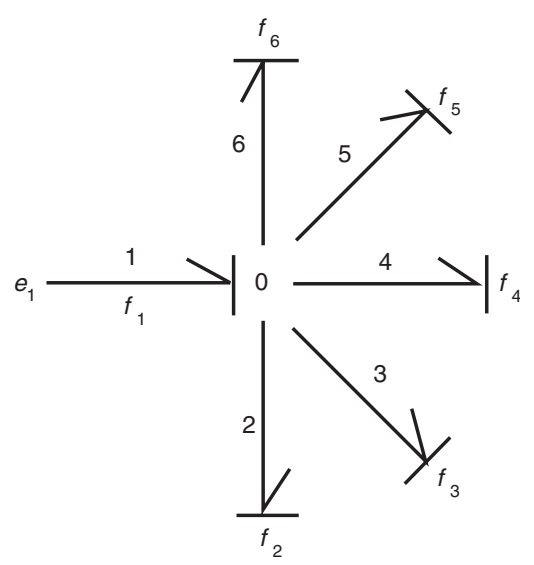

Fig. 26 0-junction

\subsection{1-Junction.}

Figure 27 shows the 1-junction. At the 1-junction, only one bond at the junction decides the flow at the junction and hence the flow at all bonds. The effort at all the bonds except the flow deciding bond decides effort at flow deciding bond. In Fig. 27, bond 3 decides the flow at all bonds and bonds 1,2 and 4 decide effort at bond 3. In 


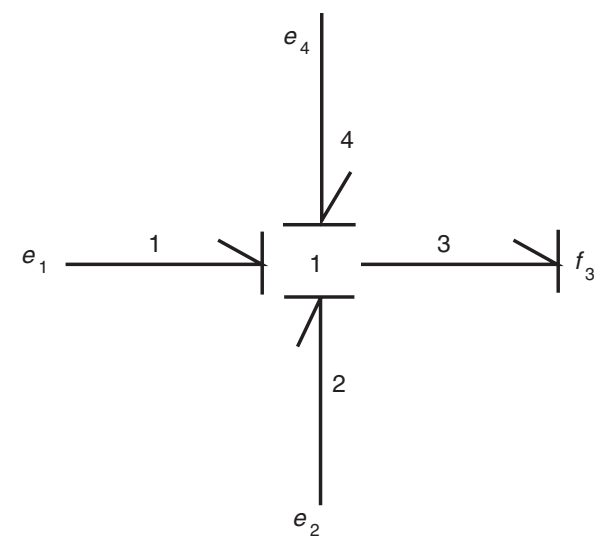

equation form as

$$
\begin{aligned}
& f_{1}=f_{3} \\
& f_{2}=f_{3} \\
& f_{4}=f_{3} \\
& e_{3}=e_{1}+e_{2}+e_{4}
\end{aligned}
$$

Fig. 27 1-Junction 